TITLE: A PHASE-MODULATION INTERFEROMETER FOR ICF-TARGET CHARACTERIZATION

AUTHOR(S): David E. Cooper

SUBMITTED TO: 28th National Vacuum Symposium Disneyland Hotel Anaheim, Californta

November 3-6, 1981

\title{
MASTER
}

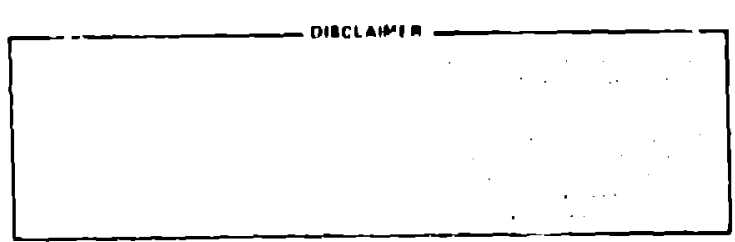

\footnotetext{
By eccoptence of inis orficle, the publisher recognires inat in US. Covernment reterns a nonexclusive, poyaliy fren licenas to publish or reprodice ine putslisted loim of this contribu lion, or to dlow orken to clo so, fur U.S. Govarmant pue 1100es

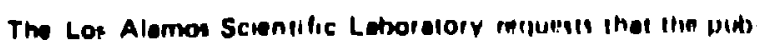
lisher identily this enliclo a work lierformad unilor the eus puces of the U.S. Deperiment of Eneroy
}

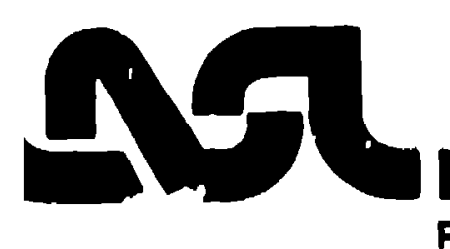




\section{A PHASE MODULATION INTERFEROMETER FOR \\ ICF TARGET CHARACTERIZATION}

$$
\begin{gathered}
\text { David E. Cooper } \\
\text { University of Californta } \\
\text { Los Alamos National Laboratory } \\
\text { P. O. Box } 1663 \\
\text { Los Alamos, NM } 87545
\end{gathered}
$$

Characterization requirements for high gain laser fusion targets are severe. We are required to Jetect defects on the surf aces of opaque and transparent shells with an amplitude resolution of $\pm 5 \mathrm{~nm}$ and a spatial resolution of 1-10 um. To achieve this we have developed a laserllluminated phase-modulation interferometer. This instrument is based on - photoelastic polarization modulation technique which allows one to convert phase information into an intensity modulation which can be easily and sensitively measured using ac signal processing techniques. This interferometer has detected path length changes as small as I nill and the required spatial resolution is assured iy using a microscope objective to focus the probe laser beam down to a smal? ( $1 \mu \mathrm{m})$ spot on the surface of a microballoon. The interferometer will soon be coupled to an LSI-11 controlled $4 \pi$ sphere manipulator which will allow us to automatically inspect the entire surface area of a target sphere. 


\section{A PHASE MODULATION INTERFEROMETER FOR \\ ICF TARGET CHARACTERIZATION}

David E. Cooper

University of California

Los itlamos National Laboratory

P. 0. Box 1663

Los Alamos, NM 87545

Character'zation requirements for high gain laser fusion targets are severe. We will soon be required to detect localized defects on the surfaces of opaque and transparent shells with an amplitude resolution of \pm $5 \mathrm{~nm}$ and a spatial resolution of 1 to 10 microns. To achieve this a novel phase shifting interferoineter has been proposed. (1) We nave constiucted a similar interferometer which uses a different modulation techn!que based on the photoelastic polarization modulator. $(2,3)$ This interfercmeter can detect $5 \mathrm{~nm}$ path length changes with a spatial resolution of $i$ to 10 microns and $c$ an examine opaque as well as transparent targets. It will soon be interfaced to an LSI-1I controlled $4 \pi$ sphere manipulator (4) which will allow us to automatically inspect the entire surface area of the torget sphere.

With conventional interferometry one can at best visually oetect a shift in an interference pattern of one-tenth of a fringe. In a double pass instrument, such as a Micheison Interferometer, this corresponds to a path length change of one-twentletı; of a wave or about $25 \mathrm{~nm}$ for visible light. This sensitivity to path length changes can be signifleant ly increased by using phase modulation interferometry. In our interferometer a phase measurement is converteo into an intensity 
measurement by sinusoldally modulating the relative phase of the two arms of the interferometer. Introduction of a phase object, such as a inicroballoon, in one arm of the interferometer results in an intensity modulation at a specific frequency on the output beam. The modulation depth is simply related to the phase shift induced by the sample object and can be eastly and sensitively measured using standard ac signal processing techniques. With this technique we have detected peth length changes as small as $1.0 \mathrm{~nm}$.

Since we are dealing with coherent, polarized light it is convenient to discuss the propagation through the interferometer in the language of the Jones calculus. (5) In this language electric fields are represented by column vectors, known as Maxwell columns, whose components are comolex numbers representing the amplitude and phase of the field. The effect of every optical element in a system can be represented by a $2 \times 2$ matrix. Multiplication of the input field vector by the representative matrix gives the output fleld from the corresponding optical element. In this language the effect of the modilator is represented by the matrix,

$$
\left[\begin{array}{ll}
1 & 0 \\
0 & e^{16}
\end{array}\right]
$$

where o - A sinwt, A is tha relasive phase amplitude and ". is the $50 \mathrm{KHz}$ fundamental longitudinal resonince of the modulator. Figure 1 1llustrates the interferometer design. Spatially flltered and expanded light from a helfum-neon laser $(632.8 \mathrm{~nm})$ is plane polarized at $+45^{\circ} \mathrm{bj}$ a 
Glan-Thompson prism. Assuming unit intensity, the light incident on the modulator is represented by the column vector $\sqrt{2}\left[\begin{array}{l}1 \\ 1\end{array}\right]$ The light output from the modulator is therefore,

$$
\frac{1}{\sqrt{2}}\left[\begin{array}{ll}
1 & 0 \\
0 & e^{i \delta}
\end{array}\right]\left[\begin{array}{l}
1 \\
1
\end{array}\right]=\frac{1}{\sqrt{2}}\left[\begin{array}{l}
1 \\
e^{i \delta}
\end{array}\right]
$$

This resulting polarization-modulated light then encounters a polarizing cube beamsplitter which transmits the $\left[\begin{array}{l}1 \\ 0\end{array}\right]$ component and ref lects the $\left[\mathrm{e}^{18}\right]$ component through $90^{\circ}$. The transmitted component becomes circularly Dolarized after passing through a suitably oriented quarterwave plate and is then focused to a small spot on the surface of a microballoon by a strain-free (0.4 N.A.) microscope objective. By changing the expansion rat to of the laser-beam expander the spot diameter can be varted from 1 to 10 microns. If the microballoci is metalific the beam is reflected at normal incidence back through the objective and waveplate. Since the light has passed through the wave-plate twice, its Dolarization state is again linear but now given by the Maxwell column $\left[\begin{array}{l}0 \\ 1\end{array}\right]$. For a transparent microbalioon one simply inserts a double-sided removable mirror at the location shown and the beam is reflected back throunh the target, objective pair and wave-plate. This probe beam is then deflected through $90^{\circ}$ on its next enccunter with the cubc beamsolitter. The $\left[\begin{array}{l}0 \\ e^{i s}\end{array}\right]$ component is also circularly polarized by a quarter-wave plate and then is retroreflected by $t$ reference mirror a second time through the wave plate becoming $\left[\begin{array}{l}\mathrm{e}^{16} \\ 0\end{array}\right]$ IIght. This reference 
beam is undeflected during its next pass through the cube beamsplitter. The probe and reference beams now travel a common path until they encounter a second cube beamsplitter. The same process is repeated with the exception that the probe beam is now focused onto the center of curvature of the target sphere (or is simply retroreflected by the removatle mirror if the target is transparent). The advantage of this is that as the sphere is rotated by the $4 \pi$ manipulator a small $( \pm 1 \mu \mathrm{m})$ mevement of the sphere along the beam axis will not change the total path length of the probe beam. (6) Otherwise, small translations of the target sphere would give rise to the same signal as a surface defect. The probe and reference beams are recombined by the beamsplitter and then pass through a Soleil-Babinet compensator and Glan-Thompson prism. The compensator introduces a relative phuse shift between the probe and reference beams which is easily adjustable to an accuracy of $5 \mathrm{~nm}$. The Glan-Thompson prism, oriented at $+45^{\circ}$, interferes the orthogonal probe and reference beams and the resulting light intensity is then detected with a photodiode. In our matrix representation, the beam coming out of the interferometer has an electric field given by $\frac{1}{\sqrt{2}}\left[\begin{array}{l}e \\ e\end{array}\right]$ where $\phi$ represents the phase shift due to any path length difference between the probe and reference arms. The effect of the compensator and polarizer is then,

$$
\begin{array}{r}
\frac{1}{2 \sqrt{2}}\left[\begin{array}{ll}
1 & 1 \\
i & 1
\end{array}\right]\left[\begin{array}{cc}
e^{-i c 1} & 0 \\
0 & e^{-i c 2}
\end{array}\right]\left[\begin{array}{l}
e^{i \phi} \\
e^{i \delta}
\end{array}\right] \\
=\frac{1}{2 \sqrt{2}}\left[e^{i(\phi-c i)}+e^{i(\delta-c 2)}\right]\left[\begin{array}{l}
1 \\
1
\end{array}\right]
\end{array}
$$


The intensity falling on the photodtode is therefore,

$$
I=\frac{1}{2}[1+\cos (\phi-\varepsilon-\delta)]
$$

where $\varepsilon=\varepsilon_{1}-c_{2}$ is the relative phase shift induced by the compensator. Expanding the cosine in equation (1) and usirig the series expansions in Besse? functions for $\cos (\delta)$ and $\sin (\delta)$ yields,

$$
\begin{aligned}
I=\frac{1}{2}\{1 & +\cos (\phi-\varepsilon) \quad\left[J_{0}(A)+\sum_{1}^{\infty} 2 J_{2 n}(A) \cos (2 n \omega t)\right] \\
& \left.+\sin (\phi-\varepsilon)\left[\sum_{0}^{\infty} 2 J_{2 n+1}(A) \sin (2 n+1) \omega t\right]\right\}
\end{aligned}
$$

In practice we detect the component in equation (2) that oscillates at the furdamental,

$$
I(\omega)=J_{1}(A) \sin (\phi-c) \sin \omega t
$$

Introduction of a phase shift $(b-c)$ between the probe and reference waves results in ali intenstty modulation at the fundamental osclllation frequency $(50 \mathrm{kHz})$ of the photoelastic modulator. This signal is detected by a lock-in amplifier referenced to the modulator.

A picture of the actual interferometer is shown in Figure 2. To obtain excellent thermal stability for the interferometer, most of ths structure was fabricated out of super-invar (thermal expanston coefficlent $\leq 3.6 \times 10^{-7}$ ). Optical elements of the interferometer rest on 
super-invar $p$ ?ates which are kinematically munted to two 1-inch-diameter super-invar rods. These rods are mounted on a super-invar fork which is in turn kinematically mounted to the optical table. To reduce the effects of floor vibration, air currents and acoustic coupling, the optical table is mounted on vibration isolation legs and both the table and interferometer are enclosed in plexiglass cages. Thermal drift in the laboratory is typically $\pm 0.5^{\circ} \mathrm{C}$ during any 8 -hour period. The effects of this drift on the interferometer are minimized by using all super-invar construction in several of the mirror mounts as well as low expansion (Zerodur) substrates for all mirrors. The physical path lengths of the probe and reference arms must only be matched to better than $0.5 \mathrm{~cm}$ since iassuming uniform thermal expansion of the super-invar framework) for each $\mathrm{cm}$ of path difference a $1^{\circ} \mathrm{C}$ temperature change will give rise to a $3.6 \mathrm{~nm}$ drift.

In an actual microballoon examination the target will be held in the interferoneter by the target rotator tips of the $4 \pi$ manipulator. To factlitate the precise positioning of the target within the interferometer a viewing optics system will be set up which provides two orthogonal views of the target. One of these views is obtained by inaking one of the common path mirrors partially transmitting as shown. After a target is loadef, the compensator will be adjusted to null out any $50 \mathrm{kHz}$ signal due to a path difference between the probe and reference beams. The sphere w1ll then be rotated throughout its $4 \pi$ solid angle by the computer-controlled manipulator and any probe path length changes brough: about by lccalized surface defects or aspherictities wtll be detected as a
\end{abstract}


$50 \mathrm{kHz}$ signal by the lock-in amplifier. In this way a detailed surface map of the sphere will be obtained.

Figure 3 shows a block diagram of the proposed automated sphere mapping system based on an LSI-11 microcomputer. A FORTRAN program will issue the commond sequence necessary to rotate the sphere under examination while simultaneously monitoring the $50 \mathrm{kHz}$ output of the lock-in amplifier. Examination times should typically be on the order of 5 minutes for a 500 micron diameter shell. After an inspection, the surface map of the sphere will be displayed on a graphics terminal and stored on magnetic disk. To facilitate comparision with other targets a hard copy of the surface map can he generated for the graphics display. 
FIGURES

Figure 1. Phase Modulation Interferometer.

Figure 2. The Phase Modulation Interferometer on Super-Invar Framework

Figure 3. Automated Sphere Mapping System.

\section{REFERENCES}

(1) J. A. Monjes, B. W. Weinstein, D. L. Willenborg, Reflection/Transmission Phase Shift Interferometer and Viewing Optics, UCRL-85281.

(2) S. M. Jasperson and S. E. Schnatterly, Rev. Sci. Inst. 40, 761-767 (1969).

(3) J. C. Kemp, J. 0pt. Soc. Am. 59, No. 8 (1969).

(4) J. A. Monjes, B. W. Weinstein, D. L. Willenborg and A. L. Richmond, Microsphere Rotator, UCRL-83375.

(5) A. Gerrard and J. M. Burch, Introduction to Matrix Methods in Optics, John Wiley and Sons (1975) pp 207-221.

(6) Laser Program Annual Report - 1979, Lawrence Livermore Latoratory, Livermore, Californta, UCRL-50021-79 (1981), Pp. 4-42 to 4-45.

(7) J. W. Berthold, S.F. Jacobs and M. A. Norton, Metrologia 13, 9-16 (1977). 


\section{PHASE MODULATION INTERFEROMETER}

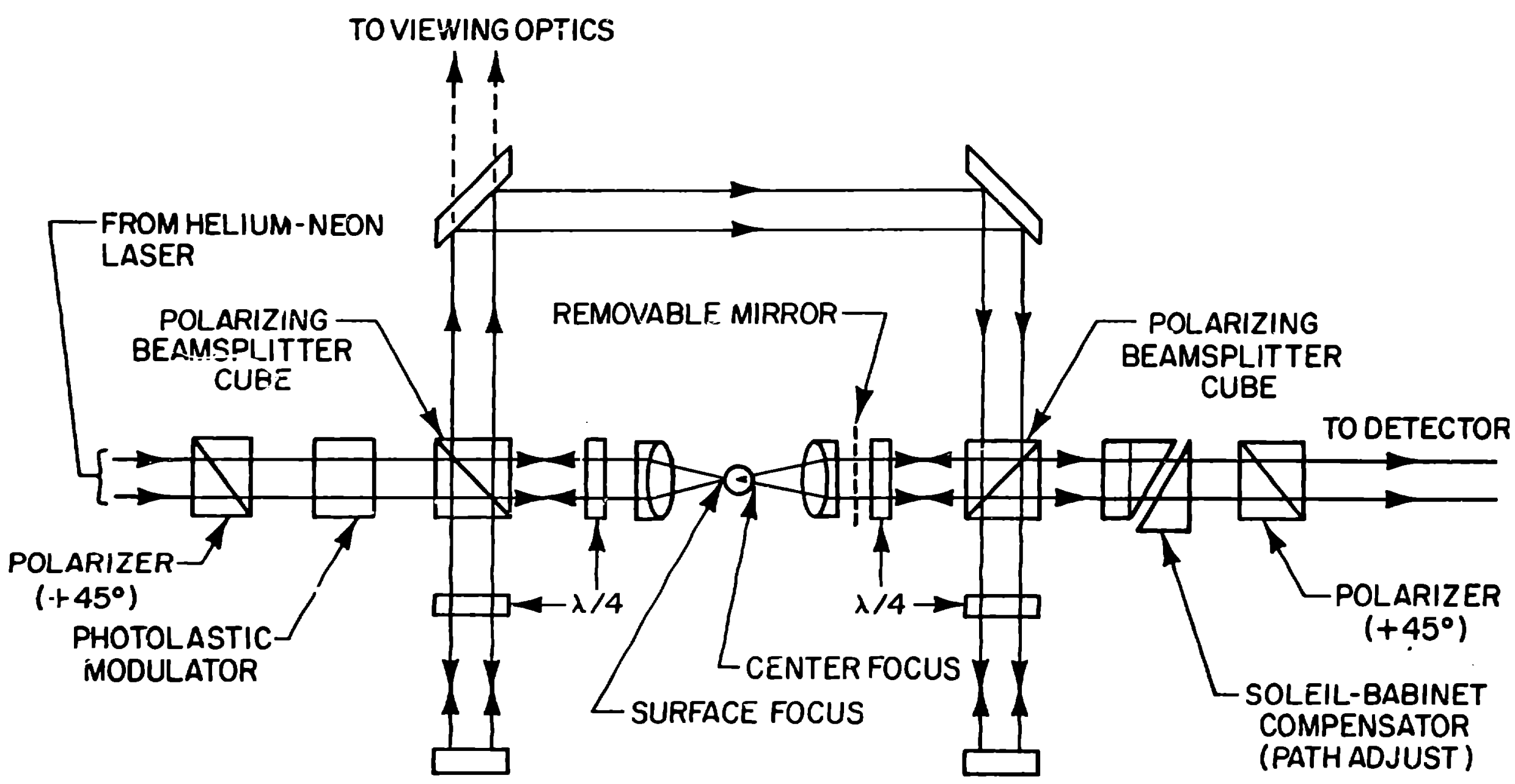




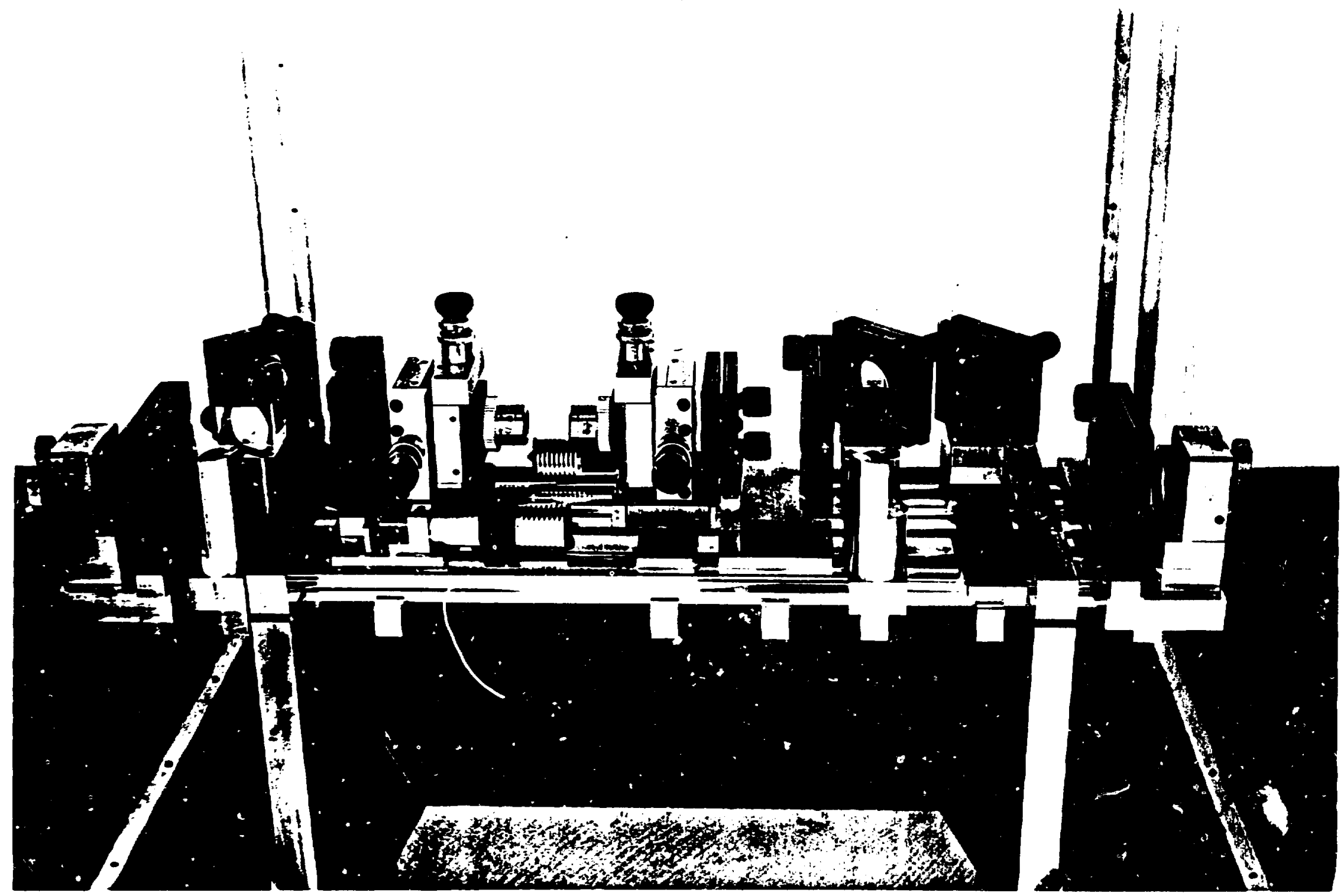


AU I UIVIAI CU STRERI IVIAT RIIVU S I I CIVI

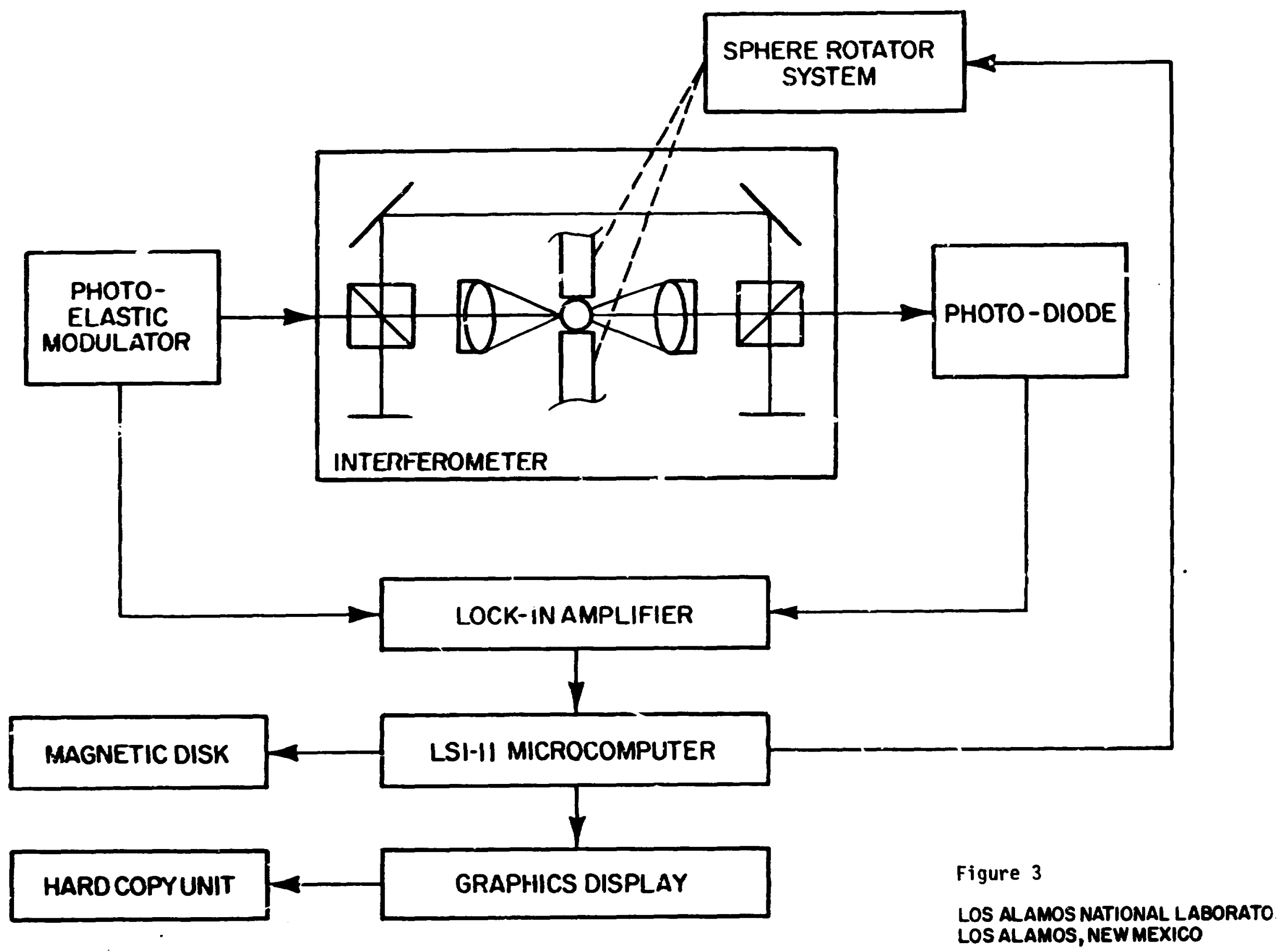

